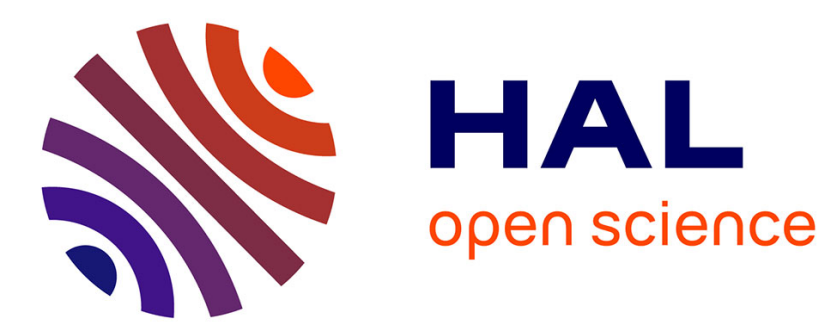

\title{
Utilisation des pulpes de betteraves sèches par les faons (Cervus Elaphus) après sevrage
}

\author{
A. Brelurut
}

\section{To cite this version:}

A. Brelurut. Utilisation des pulpes de betteraves sèches par les faons (Cervus Elaphus) après sevrage. Productions Animales, 1992, 5 (3), pp.223-228. hal-00895978

\section{HAL Id: hal-00895978 https://hal.science/hal-00895978}

Submitted on 1 Jan 1992

HAL is a multi-disciplinary open access archive for the deposit and dissemination of scientific research documents, whether they are published or not. The documents may come from teaching and research institutions in France or abroad, or from public or private research centers.
L'archive ouverte pluridisciplinaire HAL, est destinée au dépôt et à la diffusion de documents scientifiques de niveau recherche, publiés ou non, émanant des établissements d'enseignement et de recherche français ou étrangers, des laboratoires publics ou privés. 
INRA Prod. Anim., $1992,5(3), 223-228$

\section{A. BRELURUT}

INRA Laboratoire Adapatation des herbivores aux milieux Theix 63122 Saint Genès-Champanelle

\section{Utilisation} des pulpes de betteraves sèches par les faons (Cervus Elaphus)
après sevrage

L'élevage du cerf à des fins de production de viande est récent en France, et se développe simultanément dans des zones herbagères traditionnelles d'élevage -Bretagne, Normandie, Auvergne-, et dans des zones céréalières -Picardie, Centre-(Brelurut 1991). On compte aujourd'hui environ 120 élevages d'un effectif moyen de cinquante femelles en reproduction.

Dans la majorité des cas, les faons sont sevrés entre début septembre et début décembre, hivernent en bâtiment et reçoivent au cours de leur premier hiver une alimentation composée de fourrages et d'aliments concentrés (céréales seules ou en mélange, concentrés du commerce) en proportions variables. Afin de réduire le coût de l'alimentation, certains éleveurs envisagent d'utiliser des sous-produits tels que les pulpes de betteraves sous une forme de présentation simple (mélange fermier).

L'objet de cette étude était donc de tester l'utilisation de pulpes déshydratées de betteraves par des faons sevrés en comparaison

\section{Résumé}

Cette étude compare les performances hivernales réalisées par des faons mâles et femelles sevrés, recevant à volonté une ration à base de pulpes déshydratées de betterave à celles obtenues avec une ration à base de concentré complet du commerce. Les pulpes étaient complémentées par du tourteau de soja (24\% du total) et par un complément minéral et vitaminique $(1 \%)$. Ces trois composants étaient présentés sous forme de mélange fermier ou sous forme agglomérée. Les faons recevaient en outre un foin de prairie permanente de bonne qualité à volonté.

Sur l'ensemble de la période, les faons alimentés avec la pulpe ont eu un gain de poids vif total supérieur de $15 \%$ à celui des faons recevant l'aliment complet $(34,5 \mathrm{~kg}$ contre $31,1 \mathrm{~kg}$ ), mais les bois des mâles étaient un peu moins développés. Les différences ne sont toutefois pas significatives.

Les quantités ingérées d'aliments concentrés ont été plus élevées dans le cas des lots recevant la pulpe (1460 contre $1295 \mathrm{~g} \mathrm{MS} / \mathrm{faon} / \mathrm{j})$.

Les pulpes déshydratées de betterave correctement complémentées en azote et en minéraux permettent donc à des faons de réaliser des performances au moins équivalentes à celles obtenues avec un aliment complet plus coûteux. Dans nos conditions expérimentales, le mode de présentation de la pulpe (mélange fermier ou aggloméré) n'a pas eu d'effet sur les performances. d'un aliment concentré classique déjà utilisé en station expérimentale sur faons.

La forme de présentation pouvant avoir un effet sur le niveau global de consommation et sur le tri d'une partie des composants, l'aliment à base de pulpes était offert sous deux formes : soit en mélange fermier, soit en aliment aggloméré.

\section{Conditions expérimentales}

\section{1 / Principe et mise en lots}

L'expérience s'est déroulée entre le sevrage (16 septembre 1991) et la mise à l'herbe (30 mars 1992). On a utilisé 31 faons mâles et femelles, nés entre le 21 mai et le 24 juin et allaités par leur mère, entrés en bâtiment dès le sevrage et répartis en 4 lots homogènes sur les critères sexe, date de naissance, poids à la naissance et croissance pendant la phase d'allaitement (tableau 1).

Chaque lot recevait un bon foin de seconde coupe offert à volonté et l'un des aliments concentrés suivants :

- lot "pulpe fermier" : mélange pulpe + tourteau de soja + CMV, offert à volonté ;

- lot "pulpe aggloméré" : le mélange précédent sous forme agglomérée, offert à volonté ;

- lot "concentré 1 " : un aliment du commerce offert à volonté ;

- lot "concentré 2" : le même aliment concentré du commerce en quantités ajustées à la consommation du lot "pulpe aggloméré", au cas où celle-ci serait inférieure à celle du lot "concentré 1 ".

La composition minérale des pulpes étant très déséquilibrée (Hoden et al 1975) et les besoins des faons en minéraux et en oligo-éléments très mal connus (Brelurut et al 1990), nous avons offert en plus du CMV des blocs de sel à lécher supplémenté en oligo-éléments afin d'éviter tout risque de carence. 
Tableau 1. Caractéristiques des lots.

\begin{tabular}{|c|c|c|c|c|c|}
\hline Lot & $\begin{array}{l}\text { Effectif } \\
\mathrm{M}\end{array}$ & $\begin{array}{c}\text { Date de } \\
\text { naissance }\end{array}$ & $\begin{array}{l}\text { Poids à la } \\
\text { naissance } \\
\quad(\mathrm{kg})\end{array}$ & $\begin{array}{c}\text { GMQ } \\
\text { nais - sev } \\
(\mathrm{g} / \mathrm{j})\end{array}$ & $\begin{array}{c}\text { Poids de } \\
\text { sevrage } \\
(\mathrm{kg})\end{array}$ \\
\hline Pulpe fermier & 5 & $\begin{array}{c}6 \text { juin } \\
(10 \mathrm{j})\end{array}$ & $\begin{array}{c}8,4 \\
(0,8)\end{array}$ & $\begin{array}{l}331 \\
(48)\end{array}$ & $\begin{array}{l}41,1 \\
(7,6)\end{array}$ \\
\hline Pulpe aggloméré & 5 & $\underset{(9 \mathrm{j})}{8 \text { juin }}$ & $\begin{array}{c}8,4 \\
(1,0)\end{array}$ & $\begin{array}{l}339 \\
(41)\end{array}$ & $\begin{array}{l}41,9 \\
(6,8)\end{array}$ \\
\hline Concentré 1 & 5 & 2 juin & $\begin{array}{c}8,5 \\
(1,0)\end{array}$ & $\begin{array}{l}336 \\
(48)\end{array}$ & $\begin{array}{l}43,1 \\
(7,0)\end{array}$ \\
\hline Concentré 2 & 4 & $\underset{(6 \mathrm{j})}{4 \text { juin }}$ & $\begin{array}{c}8,5 \\
(1,3)\end{array}$ & $\begin{array}{l}343 \\
(47)\end{array}$ & $\begin{array}{l}43,1 \\
(7,0)\end{array}$ \\
\hline
\end{tabular}

Les écarts-types sont entre parenthèses.
Un échantillon de chacun des aliments a été prélevé une fois par semaine afin de déterminer leur teneur en matière sèche.

Les faons ont été pesés une fois par semaine pendant la période de stabulation, puis 10 jours après la mise à l'herbe.

En plus de ces mesures zootechniques classiques, la pousse des bois des mâles a été contrôlée, car elle est considérée comme un bon indicateur du niveau nutritionnel (Fennessy et Suttie 1985). Leur dimension a été enregistrée dès que leur taille était suffisante pour effectuer la mesure. Dans le cas contraire, on notait leur date d'apparition dès que leur présence était décelable au toucher.

\section{Résultats}

Les principales caractéristiques des aliproportion des différents composants des lots "pulpe" a été ajustée de façon à obtenir un aliment de mêmes valeurs énergétique et azotée que celles du concentré du commerce : $75 \%$ de pulpes, $24 \%$ de tourteau de soja et $1 \%$ de complément minéral et vitaminique (CMV). Ce CMV contenait en plus des minéraux et des vitamines deux acides aminés soufrés : lysine et méthionine.

Les aliments concentrés ont été offerts à volonté après une période de transition pendant laquelle les quantités offertes ont été progressivement augmentées de 300 grammes par jour et par faon.

Les faons ont été déparasités à deux reprises le 13 septembre et le 7 octobre (IVOMEC), et vaccinés contre l'entérotoxémie le 18 septembre et le 21 octobre (MILOXAN).

\section{2 / Mesures}

Les quantités offertes et refusées d'aliments concentrés des 4 lots ont été contrôlées chaque jour, celles des fourrages 2 fois par semaine.

\section{1 / Adaptation aux aliments}

Le diamètre plus important des granulés de pulpe et la dureté des granulés des deux aliments à base de pulpe n'ont pas posé de problème d'adaptation. La durée de la phase de transition est la même pour chacun des lots, soit 9 jours.

Durant toute l'expérience, les refus du lot "pulpe fermier" étaient exclusivement composés de pulpe, les faons consommant en priorité le tourteau de soja. En outre, le CMV n'a pas été consommé en totalité, du fait de sa présentation en semoule qui a entraîné son dépôt au fond de l'auge.

\section{2 / Etat sanitaire}

Aucun problème sanitaire ou pathologique lié à la nature des aliments n'a été relevé. Un faon du lot "concentré 2 " est mort brutalement le 22 février, probablement de yersiniose, mais le diagnostic n'a pas été confirmé par les analyses de laboratoire.

Tableau 2. Principales caractéristiques des aliments.

\begin{tabular}{|l|c|r|r|rrr|}
\hline & Forme & $\begin{array}{c}\text { Diam. } \\
(\mathrm{mm})\end{array}$ & $\begin{array}{c}\text { Teneur } \\
\text { en MS }\end{array}$ & \multicolumn{3}{|c|}{ Teneur/kg de MS (2) } \\
& & & & & & \\
\hline Composant & granulé & 10 & 88,7 & 1,01 & 63 & 106 \\
$\quad$ Pulpe déshydratée & granulé & 5 & 87,9 & 1,18 & 388 & 263 \\
Tourteau de soja & semoule & -- & -- & $\ldots$ & $\ldots-$ & $\ldots$ \\
Complément minéral & & & & & & \\
\hline Aliment & mélange & -- & 88,5 & 1,04 & 140 & 143 \\
$\quad$ Pulpe fermier & granulé & 5 & 89,5 & 1,04 & 140 & 143 \\
Pulpe aggloméré & granulé & 5 & 88,4 & 1,08 & 140 & 141 \\
Concentré complet & brin long & -- & 86,7 & 0,83 & 125 & 103 \\
Foin seconde coupe & &
\end{tabular}

(1) Moyennes observées sur l'ensemble de la période de mesure

(2) Tables INRA (1988) 


\section{3 / Consommations}

Après une première phase d'accroissement des consommations jusqu'au 15 octobre, les quantités ingérées diminuent régulièrement jusqu'au début du mois de février où elles augmentent de nouveau (figure 1).

Les aliments à base de pulpe sont globalement mieux consommés que l'aliment concentré (figure 2) : le maximum après l'entrée en bâtiment est plus élevé ( 1700 et $1750 \mathrm{~g}$ de matière sèche par faon et par jour pour les lots "pulpe fermier" et "pulpe aggloméré" contre 1630 et $1570 \mathrm{~g}$ pour les deux lots "concentré"), et le début de la phase de diminution des consommations est plus tardif (le 4 novembre contre le 14 octobre). Ce niveau plus élevé persiste jusqu'au début de la période de reprise de la consommation. Il n'a donc pas été utile de limiter les quantités offertes au lot "concentré 2", qui a été alimenté à volonté. Ses performances étant très voisines du lot "concentré 1", certains résultats sont regroupés sous l'appellation "concentré".

Globalement, sur l'ensemble de l'expérience, les faons des deux lots pulpe ont consommé 280 et $272 \mathrm{~kg}$ de matière sèche d'aliments concentrés contre 242 et $248 \mathrm{~kg}$ pour les lots concentré, soit en moyenne 1480 et $1440 \mathrm{~g}$ de matière sèche par faon et par jour, contre $1295 \mathrm{~g}$ par jour pour les faons des lots "concentré" (tableau 3). Ces derniers n'ont que partiellement compensé la différence par une ingestion légèrement accrue de fourrage : 250 $\mathrm{g}$ de $\mathrm{MS} / \mathrm{j}$ contre $200 \mathrm{~g}$ pour les faons des lots pulpe soit sur l'ensemble de la période $47,9 \mathrm{~kg}$ contre $38,3 \mathrm{~kg}$.

\section{4 / Poids vifs et variations de poids}

L'évolution du poids vif de l'ensemble des lots s'est ralentie pendant l'hiver (figure 3). La croissance des faons des deux lots pulpe qui était de $289 \pm 43 \mathrm{~g} / \mathrm{j}$ entre l'entrée en bâtiment et le 25 novembre n'a été que de $125 \pm$ $48 \mathrm{~g} / \mathrm{j}$ du 25 novembre au 3 février, puis de $210 \pm 73 \mathrm{~g} / \mathrm{j}$ de cette date à la mise à l'herbe. En ce qui concerne les lots concentré, les

Tableau 3. Consommation de matière sèche par faon pendant la phase expérimentale.

\begin{tabular}{|l|c|c|c|c|}
\hline \multirow{2}{*}{\multicolumn{1}{|c|}{ Lot }} & \multicolumn{3}{|c|}{$\begin{array}{c}\text { Ensemble de la période } \\
(\mathrm{kg})\end{array}$} & \multirow{2}{*}{$\begin{array}{c}\text { Conc. } \\
(\mathrm{g} / \mathrm{j})\end{array}$} \\
\cline { 2 - 4 } & Conc. & Foin & Total & \\
\hline Pulpe fermier & 279,6 & 40,6 & 320,6 & 1479 \\
Pulpe aggloméré & 272,0 & 36,0 & 308,0 & 1439 \\
Concentré 1 & 242,0 & 49,5 & 291,5 & 1280 \\
Concentré 2 & 248,0 & 46,3 & 294,3 & 1310 \\
\hline Moyenne & 246,0 & 47,9 & 292,8 & 1295 \\
concentrés 1 - 2 & & & & \\
\hline
\end{tabular}

Figure 1. Evolution des quantités de matière sèche totale ingérée (concentré + foin) par faon et par jour.

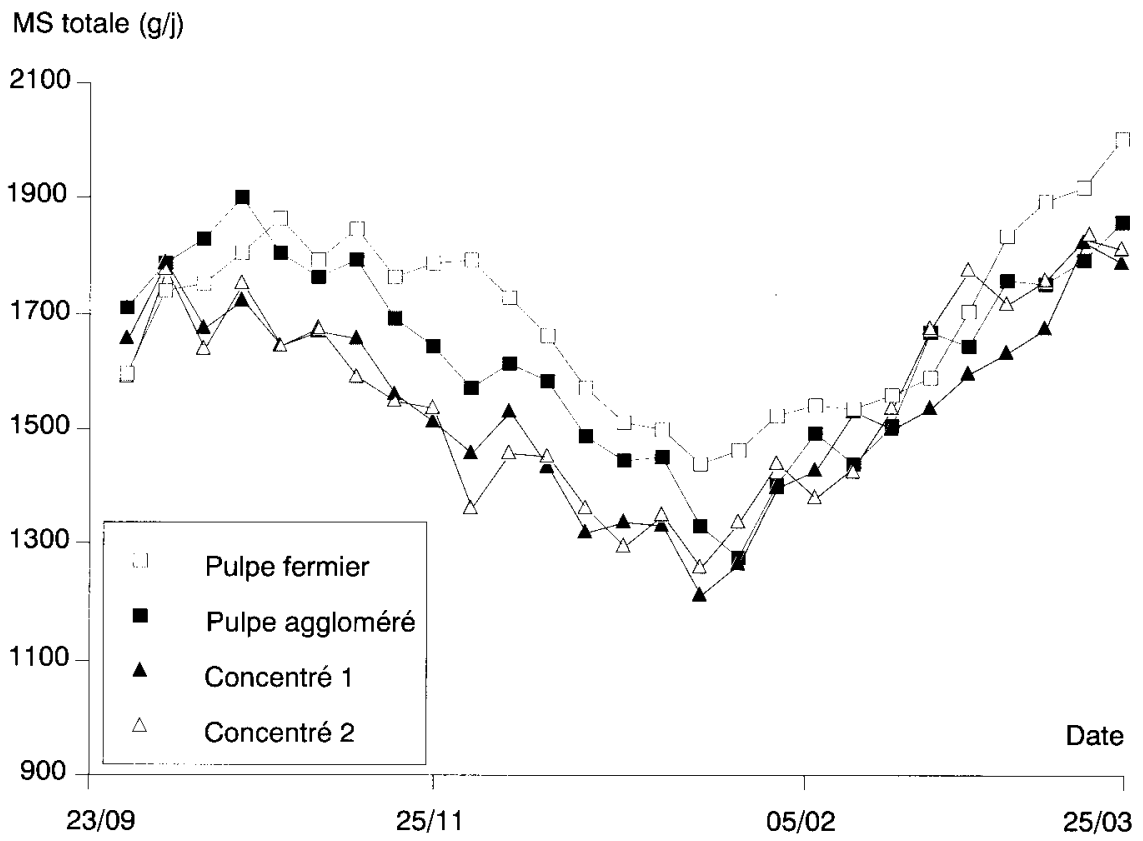

Figure 2. Evolution des quantités de concentré ingérées par faon et par jour.

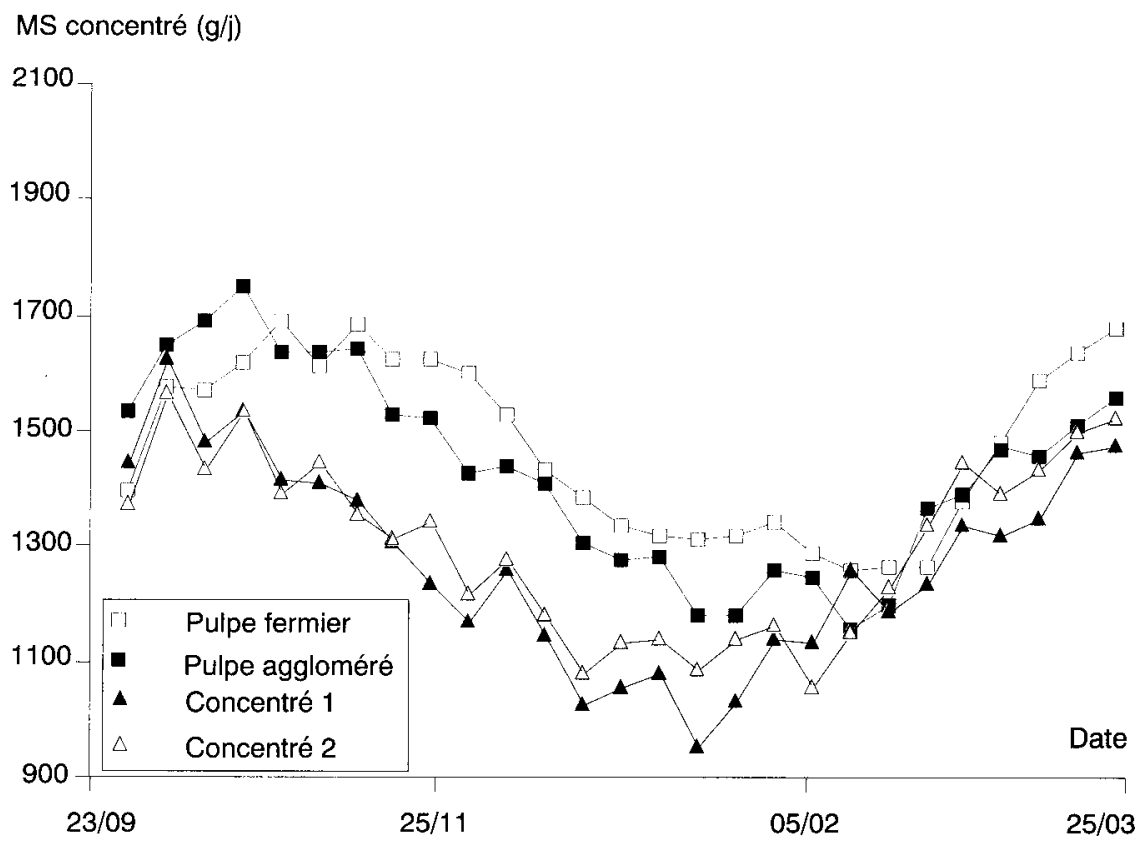

valeurs obtenues pour les mêmes périodes ont été de $238 \pm 42 \mathrm{~g} / \mathrm{j}, 122 \pm 50 \mathrm{~g} / \mathrm{j}$ et $176 \pm 52 \mathrm{~g} / \mathrm{j}$.

Le 30 mars à la mise à l'herbe, le poids moyen des faons était de 80,$4 ; 78,6 ; 77,4$ et $75,9 \mathrm{~kg}$ pour les lots "pulpe fermier", "pulpe aggloméré", "concentré 1 " et "concentré 2 ".

Pendant la période de stabulation, les faons des lots "pulpe fermier" et "pulpe aggloméré" ont gagné respectivement $38,3 \pm 7,7$ et $36,8 \pm$ $8,6 \mathrm{~kg}$ de poids vif, alors que ceux des lots "concentré" n'ont gagné que $33,3 \pm 11,0$ et $31,8 \pm 7,6 \mathrm{~kg}$, ce qui correspond à une croissance moyenne de $195,188,169$ et $162 \mathrm{~g} / \mathrm{j}$.
Le gain de poids a été un peu plus élevé pour les faons recevant la pulpe, mais la différence s'est estompée à la mise à l'herbe. 
Figure 3. Evolution du poids vif.

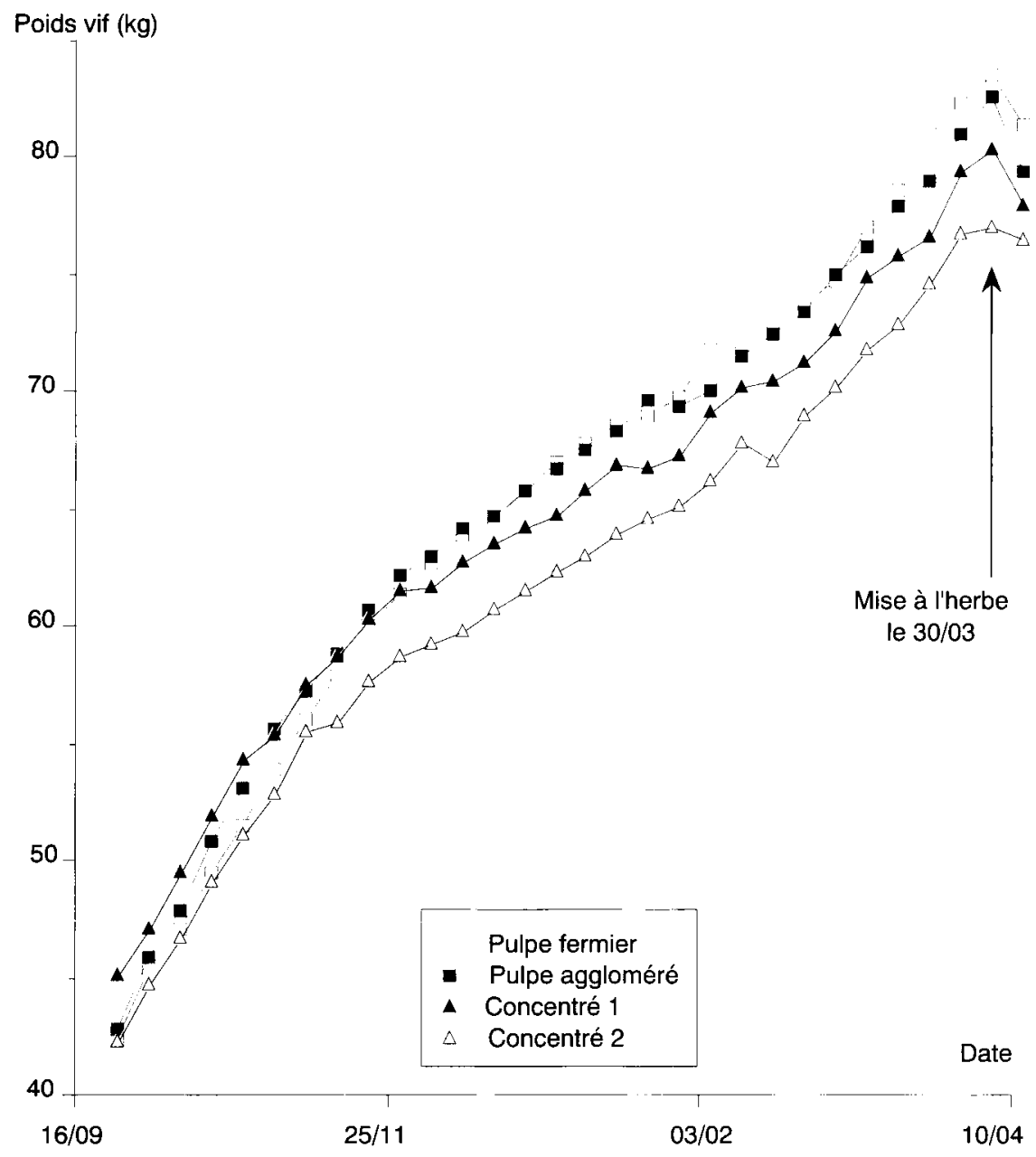

Bien que le gain total de poids vif des deux lots "pulpes" soit en moyenne supérieur de $15 \%$ à celui des deux lots "concentrés", les différences ne sont pas statistiquement différentes. Enfin, la perte de poids vif suite à la mise à l'herbe est légèrement supérieure pour les deux lots "pulpe" (2,2 et 3,5 kg contre 2,5 et $0,5 \mathrm{~kg}$ pour les lots "concentré"), ce qui réduit la différence moyenne de poids entre lots "pulpe" et lots "concentré" à $3,6 \mathrm{~kg}$.

\section{5 / Indices de consommation}

Les indices de consommation des différents lots sont voisins et varient fortement selon la période considérée : 6,15 et $6,45 \mathrm{~kg}$ de matière sèche par $\mathrm{kg}$ de gain de poids pour les lots "pulpes" et "concentré" entre le 16 septembre et le 25 novembre, 11,9 et 12,1 du 25 novembre au 3 février, et 7,9 et 7,1 du 3 février jusqu'à la mise à l'herbe.

Sur l'ensemble de la période expérimentale, la consommation en aliments concentrés par $\mathrm{kg}$ de gain de poids est légèrement plus faible pour les lots "concentré" : 7,51 et 7,20 kg de MS contre 7,55 et 7,72 pour les lots "pulpe fermier" et "pulpe aggloméré".

Si on exprime l'indice de consommation en énergie totale consommée par $\mathrm{kg}$ de gain (énergie ingérée sous forme d'aliments concentré plus celle ingérée sous forme de fourrages), on obtient un résultat inverse : 8,77 et $8,88 \mathrm{UFL} / \mathrm{kg}$ de gain dans le cas des deux lots "pulpe", et 9,08 et 9,22 UFL/kg dans le cas des lots "concentré".

Ces résultats différents selon leur mode d'expression s'expliquent par la plus forte proportion de foin dans la ration des deux lots "concentrés".

\section{6 / Croissance des bois}

La proportion de mâles des différents lots portant des bois au moins décelables au toucher à trois dates différentes, et l'évolution de leur longueur moyenne dès qu'ils ont tous été mesurables sont reportées dans le tableau 4.

La croissance des bois des mâles des lots concentré est plus rapide, et ces animaux présentent des bois plus développés que ceux des lots pulpes. Bien que la différence soit en moyenne relativement importante $(25 \mathrm{~cm}$ contre 21 en fin d'expérience), elle n'est pas significative : l'effectif est trop faible (9 et 10 mâles par groupe), et la variabilité individuelle trop importante.

\section{Discussion}

Globalement, les performances des faons sont bonnes, et les croissances sont supérieures à celle obtenues en Ecosse par Blaxter et al (1988) avec des régimes à base d'aliments concentrés. L'extrême sensibilité des cervidés au photopériodisme a été décrite par de nombreux auteurs (revue bibliographique de Thériez 1989) et elle peut expliquer cette différence car la durée d'éclairement en hiver est plus courte en Ecosse. La variation de la longueur du jour est aussi à l'origine de la diminution de la croissance et de l'appétit que nous avons observée pendant les trois mois d'hiver.

L'adaptation aux aliments à base de pulpes n'a pas posé de problèmes, contrairement aux observations faites chez d'autres espèces de ruminants, en particulier les caprins (Taccard (société USICA) communication personnelle). Toutefois, la présentation sous forme de mélange fermier a permis le tri du tourteau qui a été consommé en priorité.

Les lots "pulpe" ont eu pendant les cinq premiers mois de l'expérience une croissance plus rapide, certainement liée à une consommation plus élevée. Ces résultats sont en accord avec ceux obtenus sur agneaux par Thériez et Brun (1983), qui avaient comparé des régimes à base de pulpes déshydratées de betterave ou à base de céréales. Les agneaux recevant les pulpes avaient une consommation légèrement plus élevée (différence de $8 \%$ ), et présentaient aussi une meilleure croissance, la différence n'étant toutefois pas significative.

Chez les faons, la différence de poids à la fin de la période de stabulation qui représentait un avantage de $15 \%$, s'est toutefois réduit à 
Tableau 4. Croissance des bois.

\begin{tabular}{|c|c|c|c|c|c|c|c|c|}
\hline & \multicolumn{3}{|c|}{ Fréquence bois $\left(^{*}\right)$} & \multicolumn{5}{|c|}{ Longueur des bois (mm) } \\
\hline & $25 / 11$ & $9 / 12$ & $23 / 12$ & $13 / 1$ & $3 / 2$ & $17 / 2$ & $9 / 3$ & $23 / 3$ \\
\hline Pulpe fermier & 20 & 20 & 80 & $\begin{array}{c}2,7 \\
(2,1)\end{array}$ & $\begin{array}{c}4,9 \\
(2,9)\end{array}$ & $\begin{array}{r}7,4 \\
(3,7)\end{array}$ & $\begin{array}{l}13,9 \\
(7,6)\end{array}$ & $\begin{array}{l}19,4 \\
(8,4)\end{array}$ \\
\hline Pulpe aggloméré & 100 & 100 & 100 & $\begin{array}{c}4,6 \\
(1,9)\end{array}$ & $\begin{array}{c}7,7 \\
(3,7)\end{array}$ & $\begin{array}{l}11,4 \\
(6,4)\end{array}$ & $\begin{array}{l}18,5 \\
(8,7)\end{array}$ & $\begin{array}{l}22,6 \\
(7,1)\end{array}$ \\
\hline Moyenne pulpes & 60 & 60 & 90 & $\begin{array}{c}3,7 \\
(1,9)\end{array}$ & $\begin{array}{c}6,3 \\
(3,7)\end{array}$ & $\begin{array}{c}9,4 \\
(6,4)\end{array}$ & $\begin{array}{l}16,2 \\
(8,7)\end{array}$ & $\begin{array}{l}21,0 \\
(7,1)\end{array}$ \\
\hline $\begin{array}{l}\text { Moyenne } \\
\text { concentrés }\end{array}$ & 50 & 63 & 75 & $\begin{array}{c}7,8 \\
(6,9)\end{array}$ & $\begin{array}{l}11,7 \\
(9,4)\end{array}$ & $\begin{array}{l}14,6 \\
(9,2)\end{array}$ & $\begin{array}{l}20,6 \\
(9,6)\end{array}$ & $\begin{array}{l}25,0 \\
(9,1)\end{array}$ \\
\hline
\end{tabular}

(*) Pourcentage de mâles présentant des bois au moins décelables au toucher.

Les écarts-types sont entre parenthèses.

$4 \%$ du poids vif 10 jours après la mise à l'herbe.

Les indices de consommations varient peu d'un lot à l'autre et la valeur énergétique des pulpes de 1,01 UFL/kg de MS (INRA 1988) peut donc être retenue pour le calcul de rations destinées à des faons. Le coût énergétique d'un $\mathrm{kg}$ de gain de poids vif est cependant beaucoup plus élevé chez les faons comparativement à celui observé chez d'autres espèces de ruminants d'élevage : $23000 \mathrm{kcal}$ d'énergie métabolisable par kg de gain sur l'ensemble de la période expérimentale contre $13300 \mathrm{kcal} / \mathrm{kg}$ chez l'agneau recevant un régime comparable (Thériez et Brun 1983).

Enfin, bien que moins élevée que chez les faons des lots "concentré", la croissance des bois des mâles des lots "pulpe" n'a pas été significativement affectée.

\section{Conclusion}

L'utilisation des pulpes permet donc, quel que soit leur mode de présentation, des performances au moins équivalentes à celles obtenues avec des aliments concentrés complets, et se justifie totalement pour l'élevage du faon après sevrage.

Toutefois, certaines précautions doivent être prises par les éventuels utilisateurs (répartition en lots d'effectifs limités et longueur d'auge suffisante). En effet, le tri effectué par les faons dans le cas du mélange fermier n' a pas eu de conséquences dans nos conditions expérimentales, mais pourrait avoir des effets négatifs car la hiérarchie sociale est très marquée chez cette espèce. Les faons dominés disposent alors d'une ration éventuellement privée de certains de ses composants et risquent de réaliser des performances médiocres.

La distribution d'un aliment aggloméré semble donc préférable à celle du mélange fermier. Il est également nécessaire de supplé- menter correctement la ration en minéraux et en oligo-éléments afin d'éviter tout risque de carence.

Enfin, nous recommandons la distribution des aliments concentrés en quantités limitées : en effet, les indices de consommation très élevés ne justifient pas une alimentation à volonté, en particulier pendant la période de jours les plus courts (du 15 novembre au ler février) pendant laquelle la croissance est réduite malgré un niveau élevé d'apport énergétique. Dans le cas d'une distribution d'aliments concentrés limitée à 500 ou $600 \mathrm{~g} / \mathrm{j}$, il est nécessaire de s'assurer de la bonne qualité du fourrage offert.

Sous réserve des recommandations précédentes, l'utilisation des pulpes permet donc d'obtenir de bonnes performances. Le choix de cet aliment se justifie d'autant plus que, dans de nombreuses situations, ce produit est disponible à un prix relativement faible, et qu'il permet de remplacer de façon avantageuse les autres sources énergétiques classiquement utilisées dans les aliments complets.

\section{Remerciements}

Nous tenons à remercier l'USICA dont le soutien a permis la réalisation de cette expérience.

\section{Références bibliographiques}

BLAXTER K.L., KAY R.N.B., SHARMANN G.A.M., CUNNINGHAM J.M.M., EADIE J., HAMILTON W.J., 1988. Farming the red deer. H.M.S.O., Edinburgh.

BRELURUT A., PINGARD A., THERIEZ M., 1990. Le cerf et son élevage. Ed INRA-Le Point Vétérinaire. Paris. $144 \mathrm{p}$.

BRELURUT A., 1991. Premiers éléments sur l'élevage des cervidés en France. INRA Centre de ClermontFerrand Theix. 63122 Saint Genès Champanelle. $22 \mathrm{p}$.

FENNESSY P.F., SUTTIE J.M., 1985. Antler growth : nutritional and endocrine factors. Biology of deer production, (22) 239-250.

\section{La pulpe de betterave, complémentée en azote et en minéraux, peut être utilisée en remplacement d'un concentré classique, moyennant certaines précautions.}


HODEN A., JOURNET M., DEMARQUILLY C., GUEGUEN L., 1975. Etude de la valeur alimentaire de pulpes déshydratées. Influence de la complémentation azotée. Bull. Techn. CRZV Theix INRA (19) 5-13.

INRA, 1988. Alimentation des bovins, ovins et caprins. R. Jarrige éd. INRA, Paris.
THERIEZ M., BRUN J.P., 1983. Utilisation de pulpes de betteraves déshydratées par l'agneau à l'engraissement. Bull. Techn. CRZV Theix INRA (54) 27-30.

THERIEZ M.,1988. Elevage et alimentation du cerf (Cervus elaphus). Elevage des jeunes et production de viande. INRA Prod. Anim.,2 (2), 105-116.

\section{Summary}

\section{Use of dry beet by red deer calves after weaning}

This study compares the differences in winter growth of weaned male and female red deer calves receiving, ad libitum, a diet of dehydrated beet pulp with a ration based on a commercial com. plete concentrate.

The pulp was supplemented with soy oil-cake (24 $\%$ of the total) and with minerals and vitamins (1 $\%$ ). These three constituents were given either in the form of a farm mix or as agglomerate. 0therwise, the fawns received, ad libitum good hay of natural pasture.

Over the whole period, the fawns fed on pulp had a total live weight gain greater than $15 \%$ of that of fawns fed on complete feed (34.5 $\mathrm{kg}$ compared to
$31.1 \mathrm{~kg})$ but their antlers were less-well developed. The differences were however not significant.

The amount of concentrate ingested was higher in the case of the groups receiving the pulp $(1460 \mathrm{~g}$ as compared to $1295 \mathrm{~g} \mathrm{DM} /$ fawn/d).

The dehydrated beet pulp supplemented with nitrogen and minerals enables the fawns to gain weight in an equivalent way to those fed with more costly complete feeds. Under our experimen. tal conditions the form in which the pulp (farm mix or agglomerates) was given had no effect on performance.

BRELURUT A., 1992. Utilisation des pulpes de betteraves sèches par les faons (Cervus Elaphus) après sevrage. INRA Prod. Anim., 5 (3), 223 - 228. 